\title{
LA EXTRACCIÓN DE PRODUCTOS FORESTALES DIFERENTES DE LA MADERA EN EL AMBITO DE IQUITOS - PERÚ
}

\author{
Mauro Vásquez Ramírez * \\ Juan Baluarte Vásquez $^{* *}$
}

\section{RESUMEN}

Los productos forestales diferentes de la madera juegan un rol protagónico en la vida del poblador de la selva, sin embargo son escasamente considerados en los planes de desarrollo en comparación a otros productos que proporciona el bosque. A esta situación contribuye el escaso número de profesionales dedicados al estudio de estos recursos y la gran amplitud de productos que involucra esta clasificación. Esta realidad ha inducido a realizar una prospección de los productos que se extraen en el ámbito de la Unidad Forestal y Fauna .Iquitos, con el propósito de conocer el número de extractores que se dedican a esta actividad, procedencia de los productos que se expenden en Iquitos y los procedimientos que se utilizan para la extracción de los principales productos. Este estudio ha permitido conocer que en Iquitos se extraen 62 especies que proporcionan productos forestales diferentes de la madera. La mayor parte procede de poblaciones silvestres cercanas a poblados asentados en las márgenes de los ríos Amazonas, Nanay y sus tributarios. El escaso número de extractores registrados en los archivos del sector agrario, revela la existencia de una actividad altamente informal. La mayor parte de los extractores se dedican al aprovechamiento de irapay $(54,4 \%)$ y el producto con mayor volumen de producción controlado corresponde a palmito de huasai. La extracción de los productos forestales diferentes de la madera se realiza de diversas formas, dependiendo de la especie, usos, época del año, lugar y parte de la planta que se aprovecha.

Palabras clave: $\quad$ Extracción, productos forestales diferentes de la madera, tamshi, lmambé, shapaja. yarina, chambira, piasaba, ojé, bombonaje, Amazonía Peruana. 


\begin{abstract}
The forestry products other than lumber have an important role in the life of the inhabitant of the jungle eventhough they are hardly considered in the plans for development. Added lo this fact is the few professionals who are focusing on these resources and the great variety of products this classification comprises. This reality has motivated the making of an extensive view of the products that are extracted in the Unidad Forestal y Fauna .Iquitos, with the purpose of finding out the number of extractors that are dedicated to this activity; the source of the products that are sold in Iquitos and the procedures used for the extraction of the main items. This study has helped find out that 62 species other than wood are extracted in Iquitos, most of them coming from wild areas in the neighborhood of small towns on the Amazon, Nanay rivers and their affluents. The few registered extractors in the files of the agrarian sector tells about on activity which is highly informal. Most of them exploit the irapay (54\%) which is among the controlled products with the highest production corresponding to palmito of huasai. The extracting of products other than wood is done in different ways, depending on the species, uses, time of year, place and the parts of the plant which is used.
\end{abstract}

Key words: $\quad$ Extraction, forestry products other than wood, Peruvian Arnazon Region.

\title{
1. INTRODUCCION
}

La actividad forestal en el ámbito de la jurisdicción de la ciudad de Iquitos es una de las de mayor importancia en la vida del poblador rural, pues el bosque le proporciona una gran variedad de productos que son utilizados como alimentos, medicinas y construcción de viviendas y otros que son utilizados para diversos fines.

La mayor parte de estos productos la constituyen los denominados productos forestales diferentes de la madera, llamados también injustamente productos secundarios del bosque; estos productos contribuyen con más del $50 \% 1$ ingreso monetario a muchas familias de la región (Padoch, 1990). No obstante su importancia, por años han sido marginados de los planes de desarrollo en la región; sin embargo, a partir de esta década comienzan a despertar interés de investigadores y planificadores, publicándose algunos estudios como los desarrollados por Padoch (1990), Tello et. al. (1990), Mejía y Rengifo (1995) y 
Mejía (1992, 1995). Este último expone brevemente el estado actual de los conocimientos sobre estos productos en la región.

La gran diversidad de especies que se comercializan bajo esta denominación, ha sugerido la sistematización e interpretación de la información registrada en el Ministerio de Agricultura.

Con el objeto de acceder a la mayor información posible, se visitó algunos centros de expendio ubicados en el ámbito de Iquitos y se entrevistó a los extractores y comerciantes de estos productos.

La cantidad de productos forestales diferentes de la madera es mayor de lo que las estadísticas oficiales indican y de lo que muchos afirman; por ello es necesario acercarse más a estas especies para incorporar un mayor número a la economía local, regional y nacional.

\section{MATERIALES Y METODOS}

\subsection{Jurisdicción y características de la zona donde se realizó el trabajo}

El presente estudio se realizó en el ámbito de la Unidad Forestal y Fauna de Iquitos del Ministerio de Agricultura, comprendido dentro de los distritos de Iquitos y Punchana.

Ambas ciudades pertenecen a la provincia de Maynas, región Loreto, enmarcadas dentro de las coordenadas geográficas $30^{\circ} 45^{\prime}$ Latitud Sur y $73^{\circ} 15^{\prime}$ longitud Oeste, y a una altitud de $126 \mathrm{msnm}$. Presentan un clima con las siguientes características: precipitación media anual, $2876,5 \mathrm{~mm}$; temperatura media $24,3^{\circ} \mathrm{C}$ y humedad relativa promedio mensual, $80 \%$.

\subsection{Método}

El levantamiento de la información se efectuó revisando los registros estadísticos de la Unidad Forestal y Fauna - Iquitos; dialogando con extractores y comerciantes y mediante el seguimiento en centros de expendio.

Las zonas y contratos de extracción, así como la procedencia de los productos forestales no madereros se obtuvieron mediante la revisión de los archivos de la Unidad Forestal y Fauna .Iquitos en el periodo de seis años (1987-1992). 
Para obtener información adicional y los procedimientos de extracción que se utilizan, se visitó los centros de expendio, donde se dialogó con algunos extractores. También se revisó información bibliográfica sobre los usos que se destinan a algunos productos.

El procesamiento de la información se realizó utilizando el Programa Word Perfect

6.0 .

\section{RESULTADOS}

\subsection{Especies de productos forestales diferentes de la madera}

En el Cuadro 1 del anexo se alcanza la relación de especies que proporcionan productos forestales no maderables que se extraen en el ámbito de la ciudad de Iquitos.

\subsection{Número de extractores}

En el Cuadro 2 se indica el número de extractores registrados por la Unidad Forestal y Fauna .Iquitos, en un período de seis (06) años (1987-1992). En dicho cuadro se aprecia que en el citado periodo se registraron 180 extractores formales, correspondiendo 68, la mayor cantidad, al primer año y 10 al último. 
Cuadro 2. Número de extractores por productos forestales diferentes de la madera, período (1987 - 1992).

\begin{tabular}{|l|c|c|c|c|c|c|c|}
\hline PRODUCTO & 1987 & 1998 & 1989 & 1990 & 1991 & 1992 & TOTAL \\
\hline IRAPAY & 34 & 14 & 24 & 13 & 9 & 4 & 98 \\
AMPIHUASCA & 15 & 4 & 3 & & & & 22 \\
AGUAJE & 8 & 4 & 1 & 3 & 1 & 1 & 18 \\
PLANTAS ORNAMENTALES & 1 & & 1 & & & & 2 \\
PALMITO & 6 & & 4 & 4 & 4 & & 18 \\
PLANTAS MEDICINALES & 2 & & 1 & 1 & 1 & 4 & 11 \\
OJE & 2 & 1 & 1 & 1 & 1 & & 6 \\
HUAMBRE & & & 1 & & & & 1 \\
PIJUAYO & & & & & & & 2 \\
CORTEZA (VARIAS) & & & & 2 & 2 & & 2 \\
LATEX (VARIOS) & & & & & & 1 & 1 \\
& & & & & & & 18 \\
\hline TOTAL & 68 & 23 & 36 & 25 & 18 & 10 & 180 \\
\hline
\end{tabular}

Fuente: Estadisticas Oficiales de la Unidad Forestal y Fauna-Iquitos

\subsection{Procedencia de los productos forestales diferentes de la madera}

La mayor parte de los productos forestales diferentes de la madera que se extrae en el ámbito de la Unidad Forestal y Fauna .Iquitos, procede de plantas en estado silvestre, ubicadas en los bosques cercanos a poblados asentados en los ríos Amazonas, Nanay y sus tributarios (Cuadro 3). 
Cuadro 3. Procedencia de los productos forestales diferentes de la madera, según contratos de extracción emitidos por la Unidad Forestal y Fauna-Iquitos.

\begin{tabular}{|c|c|c|c|}
\hline PRODUCTO & RIO PRINCIPAL & TRIBUTARIO & AFLUENTE \\
\hline IRAPAY & $\begin{array}{l}\text { Nanay } \\
\text { Nanay } \\
\text { Nanay } \\
\text { Nanay } \\
\text { Nanay } \\
\text { Nanay } \\
\text { Nanay } \\
\text { Nanay } \\
\text { Nanay } \\
\text { Nanay } \\
\text { Nanay } \\
\text { Nanay } \\
\text { Amazonas } \\
\text { Amazonas } \\
\text { Amazonas } \\
\text { Amazonas } \\
\text { Amazonas } \\
\text { Nanay }\end{array}$ & $\begin{array}{l}\text { Pintuyacu } \\
\\
\text { Pintuyacu Rio } \\
\text { Chambira } \\
\\
\\
\text { Nanay } \\
\text { Nanay } \\
\text { Nanay } \\
\text { Nanay } \\
\text { Nanay } \\
\text { Pintuyacu }\end{array}$ & $\begin{array}{l}\text { Quebrada Chonta } \\
\text { Río Chambira } \\
\text { Quebrada Miranda } \\
\text { Quebrada Retama } \\
\text { Santa Rosa } \\
\text { Quebrada Flores } \\
\text { Quebrada Fortuna } \\
\text { Pto. Tipishca } \\
\text { Qbda. Entra si Puedes } \\
\text { Quebrada Patu Yacu } \\
\text { Quebrada Cascabel } \\
\text { Quebrada Francia } \\
\text { Quebrada Palometa } \\
\text { Quebrada Agua Blanca } \\
\text { Río Pintuyacu } \\
\text { Quebrada Otorongo } \\
\text { Quebrada Callao } \\
\text { Quebrada Otorongo }\end{array}$ \\
\hline AMPIHUASCA & $\begin{array}{l}\text { Nanay } \\
\text { Amazonas } \\
\text { Nanay } \\
\text { Amazonas } \\
\text { Amazonas } \\
\text { Amazonas }\end{array}$ & $\begin{array}{l}\text { Tamishiyacu Pintuyacu } \\
\text { Yarapa }\end{array}$ & $\begin{array}{l}\text { Quebrada Jazmín } \\
\text { Quebrada Supay Caño } \\
\text { Quebrada Sahua } \\
\text { Quebrada Pelaje } \\
\text { Quebrada Acarnanillo } \\
\text { Quebrada Yanayaquillo }\end{array}$ \\
\hline OJE & $\begin{array}{l}\text { Amazonas } \\
\text { Amazonas }\end{array}$ & $\begin{array}{l}\text { Yarpara } \\
\text { Yarapara }\end{array}$ & $\begin{array}{l}\text { Quebrada Pumaycu } \\
\text { Qubrada Cumaceba }\end{array}$ \\
\hline PALMITO & $\begin{array}{l}\text { Amazonas } \\
\text { Amazonas } \\
\text { Amazonas } \\
\text { Amazonas } \\
\text { Amazonas } \\
\text { Amazonas } \\
\text { Amazonas } \\
\text { Amazonas } \\
\text { Amazonas } \\
\text { Amazonas }\end{array}$ & $\begin{array}{l}\text { Arnpiyacu } \\
\text { Yarapa } \\
\text { Yarapa } \\
\\
\text { Yarapa } \\
\text { Yarapa } \\
\text { Yarapa } \\
\text { Ampiyacu }\end{array}$ & $\begin{array}{l}\text { Quebrada Sábalo } \\
\text { Quebrada Curayari } \\
\text { Quebrada Sábalo } \\
\text { Quebrada Apayadu } \\
\text { Rio Arnpiyacu } \\
\text { Quebrada Palometa } \\
\text { Quebrada Cumaceba } \\
\text { Quebrada Sabalillo } \\
\text { Quebrada Yanayacu Grande } \\
\text { Quebrada Yahuayacu }\end{array}$ \\
\hline
\end{tabular}




\begin{tabular}{|c|c|c|c|}
\hline PRODUCTO & RIO PRINCIPAL & TRIBUTARIO & AFLUENTE \\
\hline HUAMBRE & Amazonas & Tamshiyacu & Quebrada Shanabo \\
\hline AGUAJE & $\begin{array}{l}\text { Amazonas } \\
\text { Amazonas } \\
\text { Amazonas } \\
\text { Amazonas } \\
\text { Amazonas } \\
\text { Amazonas }\end{array}$ & $\begin{array}{l}\text { Yarapara } \\
\text { Yarapara }\end{array}$ & $\begin{array}{l}\text { Quebrada San Sebastián } \\
\text { Quebrada Yanayacu } \\
\text { Quebrada Corrientes } \\
\text { Quebrada Yacapana } \\
\text { Quebrada Ayacucho } \\
\text { Quebrada Yanayaquillo }\end{array}$ \\
\hline $\begin{array}{l}\text { SEMILLAS DE } \\
\text { PALMERAS } \\
\text { VARIAS } \\
\end{array}$ & Amazonas & Nanay & Quebrada Tarapoto \\
\hline $\begin{array}{l}\text { PLANTA } \\
\text { ORNAMENTALES }\end{array}$ & Amazonas & Pintuyacu & Quebrada Piñuisqui \\
\hline $\begin{array}{l}\text { PLANTA } \\
\text { MEDICINALES }\end{array}$ & $\begin{array}{l}\text { Nanay } \\
\text { Napo } \\
\text { Napo } \\
\end{array}$ & Pintuyacu & $\begin{array}{l}\text { Quebrada Casho } \\
\text { Quebrada Llanllpa } \\
\text { Quebrada Sucusari } \\
\end{array}$ \\
\hline $\begin{array}{l}\text { CORTEZAS RAICES } \\
\text { Y OTROS }\end{array}$ & Amazonas & Nanay & \\
\hline LATEX (VARIOS) & $\begin{array}{l}\text { Amazonas } \\
\text { Amazonas } \\
\text { Nanay } \\
\text { Nanay }\end{array}$ & $\begin{array}{l}\text { Nanay } \\
\text { Nanay } \\
\text { Nanay } \\
\text { Pintuyacu } \\
\text { Pintuyacu }\end{array}$ & $\begin{array}{l}\text { Río Pintuyacu } \\
\text { Quebrada Paujil } \\
\text { Quebrada Paujil } \\
\text { Quebrada Cetico } \\
\text { Quebrada Puma } \\
\text { Quebrada Salvatierra }\end{array}$ \\
\hline
\end{tabular}

Fuente: Estadísticas Oficiales de la Unidad Forestal y Fauna - Iquitos.

\subsection{Volumen de extracción de los principales productos forestales diferentes de la madera}

En el Cuadro 4 se puede apreciar el volumen de extracción controlada de los principales productos forestales diferentes de la madera, registrados en la Unidad Forestal s' Fauna Iquitos en el período de seis años (1987-1992). De acuerdo con esta información, el producto que más se aprovecha del bosque corresponde al palmito de huasaí, mientras que en el extremo opuesto está el pijuayo. 
Cuadro 4. Producción Controlada de los principales productos forestales diferentes de la madera que se extraen en el ámbito de Iquitos. (años 1987-1992)

\begin{tabular}{|c|c|c|c|c|c|c|c|c|}
\hline \multicolumn{2}{|l|}{ PRODUCTOS } & \multicolumn{6}{|c|}{$A \quad \tilde{N} O S$} & \multirow[t]{2}{*}{ TOTAL } \\
\hline & & 1987 & 1988 & 1989 & 1990 & 1991 & 1992 & \\
\hline HUA & $(\mathrm{kg})$ & 234 & 90833 & 11213 & 178799 & 380402 & 302024 & 1129475 \\
\hline & $(\mathrm{kg})$ & 230 & 0 & 200 & 4350 & 900 & & 63100 \\
\hline IRAPAY & $(\mathrm{kg})$ & 33839 & 76750 & 59800 & 57450 & 57150 & 38925 & 323914 \\
\hline AMPIHUASCA & $(\mathrm{kg})$ & 86738 & 51540 & 15967 & 1110 & $\cdots$ & -..- & 155355 \\
\hline P. MEDICINALES & $(\mathrm{kg})$ & --. & 186 & 260 & - & - & 144 & 598 \\
\hline OJE & $(\mathrm{kg})$ & 10395 & 11852 & 8418 & 9925 & 223 & 3600 & 44413 \\
\hline S. DE GRADO & (lt) & -...- & 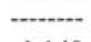 & -..- & 370 & 2490 & 2930 & 5790 \\
\hline HUAMBE & $(\mathrm{kg})$ & (-).-. & 1140 & 50 & (-) & (n) & & 1190 \\
\hline CORTEZAS & $(\mathrm{kg})$ & -...- & …… & -no- & 2215 & 1116 & 128 & 3459 \\
\hline PIJUA & $(\mathrm{kg})$ & (-)...- & - & $\cdots$ & - & 200 & $+\cdots$ & 200 \\
\hline PIASABA & $(\mathrm{kg})$ & $\cdots$ & - & -no- & 8000 & - & - & 8000 \\
\hline
\end{tabular}

Fuente: Estadísticas Oficiales de la Unidad Forestal y Fauna -Iquitos. (1993)

\subsection{Proceso de extracción}

\subsubsection{Extracción de lianas:}

\section{- Tamshi:}

Es una hemiepífita que se desarrolla en terrenos de alturas y bajiales. El tarnshi de altura es de mejor calidad porque la fibra es más fina. Esta planta trepa a cualquier especie de árbol, especialmente en aquellos que tienen un fuste más prominente. Se encuentran en asociación con la itininga.

La extracción se realiza jalando la raíz y envolviéndola en rollos, luego se vuelve a jalar la raíz repitiendo esta operación hasta donde sea posible; cuando la raíz opone resistencia, se jala con fuerza hasta romperse en un segmento que luego es enrollado y transportado hasta el lugar de expendio. Cada rollo está compuesto por raíces de 20-3 0 metros de longitud aproximadamente. Una carga está conformada por alrededor de diez rollos. 


\section{- Huambé:}

Es una hemiepifita. Sc encuentra trepando cualquier especie, preferentemente en las de mayor vigor; su raíz tiene una longitud de 15-20 metros aproximadamente (longitud aprovechable). Se encuentran formando gremios (manchales).

La forma de la raíz es redonda, con un diámetro promedio de $3 \mathrm{~cm}$ aproximadamente. Contiene una resma irritante que al hacer contacto con la piel ocasiona escozor.

La extracción se realiza cortando la base de la raíz, procediendo de manera similar a la extracción del tamshi. Una carga de huambé está conformada de cinco a diez rollos, dependiendo del diámetro de la raíz.

Esta planta puede encontrarse en zonas de altura o de bajiales, ocurriendo con mayor frecuencia en zonas bajas, formando manchales.

Para su utilización se descorteza primero la raíz, dejando al descubierto el material leñoso, el cual se divide en 5-6 secciones por cada raíz; luego es secado. Se usa para confección de canastas, carteras, acabado de sillas y otros muebles.

\subsubsection{Extracción de palmeras}

\section{- Shapaja:}

Es una palmera que proporciona frutos una vez al año. Se comercializa las boj as en grandes volúmenes, principalmente en los poblados del río Ucayali.

Esta palmera se encuentra en terrenos de altura, en las faldas de las colinas.

La extracción de las hojas se realiza cortando los pecíolos. Antes de extraerlos, primeramente se tiene que verificar el estado de las hojas (sí son maduras o no); cuando una hoja está madura, es ancha y de color verde oscuro; mientras que cuando son inmaduras, éstas son angostas y presentan color verde claro.

Las hojas maduras se emplean para el techado de casas, el endospermo de las semillas es comestible y las hojas jóvenes se aprovechan como chonta. 


\section{Yarina:}

De esta palmera se aprovechan las hojas (para techos) y el endospermo inmaduro de las semillas, que es comestible. El tiempo de duración de las hojas puestas en obra (techado) es menor, debido a que el segmento de hoja es más delgado.

La extracción se realiza sin talar la palmera, ya que la altura que alcanza es de 4-5 metros.

\section{- Chambira:}

Es una palmera con espinas. Las fibras se usan en trabajos de artesanías, como hamacas, shicras, collares y otros, mientras que el endospermo inmaduro de los frutos es comestible. Esta planta puede alcanzar hasta 20 metros de altura.

La extracción de la fibra se obtiene de los segmentos frescos de las hojas jóvenes que aún no se han desplegado. Las tiras son convertidas en cuerdas que sirven para la manufactura de diversos artículos artesanales.

\section{- Piasaba:}

Es una palmera que mide de 5-10 m de altura. Se aprovecha su fibra, que es de color negro, cuya longitud aproximada es de 5-7 metros.

Las fibras son las que cubren el peciolo y la vaina de las hojas que crecen en todo el contorno de la planta. Para extraerlas se cortan las hojas en la misma base, permitiendo así que se desprendan en capas.

Las fibras se utilizan para confeccionar escobas y cepillos, las hojas para techado de casas y las semillas son comestibles.

\subsubsection{Extracción de leche de ojé (látex)}

Para la extracción del látex de ojé, se aprovechan todos los árboles sin tener en cuenta el diámetro; pero es más recomendable el aprovechamiento en árboles jóvenes, que oscilan entre 10-12 años de edad, porque se considera que es la etapa en que los árboles almacenan mayor cantidad de exudado.

Para extraer el látex de ojé se «rasquetea» (hacer incisiones) alrededor del árbol; el distanciamiento entre cada incisión debe ser de aproximadamente 3 metros.

El volumen de látex por árbol joven es de diez litros aproximadamente. 


\subsubsection{Extracción del bombonaje}

El bombonaje se desarrolla en tierra franco arenosa, la altura que alcanza es de 3 metros y se encuentran formando gremios.

La extracción se realiza talando toda la planta, que incluye el peciolo, hojas maduras y hojas jóvenes teniendo cada uno diferente uso. El pecíolo en tiras sirve para la confección de canastas y otras artesanías, las hojas maduras para techado de casas y las hojas jóvenes en estado fresco proporcionan fibras para confeccionar diferentes tejidos (sombreros, cedazos y otras artesanías); también, luego de un proceso de cocción de las fibras (llamada fibra de «toquilla», en la región de San Martín), se procede a tejer sombreros, cedazos y otras artesanías de mayor calidad.

\section{DISCUSION}

\subsection{Especies que producen productos forestales diferentes de la madera}

El Cuadro 1 nos muestra la gran variedad de especies forestales que proporcionan una diversidad de productos como frutos, cortezas, semillas, hojas, raíces, látex, fibras y otros; algunas especies proporcionan varios productos a la vez.

La mayor parte de las especies proporciona alimentos (37\%) en forma de frutas y semillas comestibles o de hojas tiernas, el $27 \%$ provee medicinas en forma de cortezas, raíces, hojas y exudados; el 15\% suministra materias primas para artesanías. Asimismo, el $21 \%$ se destina para otros usos, ya sea para construcción de viviendas, canoas, etc. Cerca de ocho especies tienen hasta dos usos cada una.

De las 62 especies que se indican en el Cuadro 1, solamente el 20\% es controlado por el sector agrario; el resto se extrae informalmente.

Existen además otras especies forestales que son extraídas clandestinamente de nuestros bosques, que no logramos anotar y que no están registradas en las estadísticas del sector agrario, por tal motivo no han sido consideradas en este estudio.

Sin embargo, la información que se alcanza en el Cuadro 1 es suficientemente indicativa de la gran diversidad biológica que congregan los productos forestales no maderables. 
Las especies que tienen costumbres gregarias son las que se aprovechan en mayor cantidad, entre ellas tenemos: bijao, irapay, tamshi y huambé.

Es necesario resaltar que el presente trabajo se ha limitado a recabar información del sector agrario y a entrevistas a los extractores, no siendo posible verificar in situ las especies; por tal motivo no se ha colectado muestras botánicas de los especimenes, razón por la cual la taxonomía de las plantas muestra algunas incorrecciones.

\subsection{Número de extractores}

La extracción de productos forestales diferentes de la madera se viene efectuando informalmente en nuestra región desde tiempo atrás; el sector agrario consciente de la trascendencia de estos productos, los ha incorporado últimamente en sus registros de control.

En el año 1987 se registraron 68 extractores, disminuyendo progresivamente los siguientes años, hasta que en el año 1992 se registraron solamente 10 de ellos. Sin embargo, en la práctica, existe aún un gran número de extractores de estos productos que desarrollan su actividad ilegalmente y que no están inscritos en los padrones del sector agrario.

La inclusión de algunos derechos por contratos de extracción de estos productos, además de los precios subvaluados que se pagan por la venta de la materia prima, genera exiguas ganancias. En ese contexto los extractores prefieren trabajar en la informalidad.

\subsection{Procedencia de los productos forestales diferentes de la madera}

La extracción de los productos forestales diferentes de la madera está mayormente caracterizada por la utilización de plantas de poblaciones silvestres cercanas a

poblados asentados en las márgenes de los ríos, principalmente del Amazonas, Nanay y sus tributarios. Solamente el $10 \%$ de las especies tratadas proceden de cultivos antrópicos.

Toda la producción procedente de estos lugares converge en Iquitos, por ser el mercado que absorbe toda la producción de su área de influencia. 
De acuerdo con esta información, podemos afirmar que algunas especies muestran cierta preferencia hacia un área geográfica determinada; así, por ejemplo, el irapay crece mayormente en bosques de la cuenca del río Nanay, mientras que el huasaí (palmito) y el aguaje proceden generalmente de los bosques ribereños al río Amazonas.

Muchos productos que se extraen de la zona del Ucayali no llegan a Iquitos, puesto que el transporte es muy oneroso, influyendo en el costo de los productos; mayormente éstos son comercializados en Pucallpa, Contamana y Requena. En el caso de los productos que son extraídos del Marañón y Huallaga, algunas veces se comercializan en la ciudad de Nauta e Iquitos, pero en su mayoría son trasladados a Yurimaguas para luego ser distribuidos a otros lugares, entre ellos la Región San Martín.

\subsection{Volumen de extracción de los principales productos forestales diferentes de la madera}

El producto forestal diferente de la madera que más se extrae en el área de influencia del presente estudio, en términos de volumen extraído, corresponde al palmito de huasaí. En el periodo evaluado se aprecia un incremento considerable de la extracción de este producto, tendencia también apreciada por Tello et al. (1990). La población de Iquitos consume el palmíto en estado natural, acentuándose más su consumo en los días centrales de la Semana Santa. Asimismo, en Iquitos está instalada una planta envasadora de palmitos cuya producción está mayormente orientada al mercado internacional.

Otro producto que tiene buena aceptación en Iquitos es el irapay, cuyo uso está orientado a satisfacer las necesidades de vivienda de la población de escasos recursos económicos. El aprovechamiento de este producto se mantiene casi estable a través de los años. El análisis de los contratos de extracción ha permitido visualizar que un mayor número de extractores se dedica al aprovechamiento de este producto, constituyendo el $54 \%$ de los extractores oficialmente registrados.

El arnpihuasca y el aguaje son otros productos que también han tenido importante demanda por parte de los extractores; sin embargo, en los últimos años no figuran en las estadísticas del sector agrario, lo que es preocupante ya que su comercialización continúa en los principales mercados de Iquitos, siendo su consumo evidente. 
La extracción del látex de ojé permanece estable en el período evaluado, con una ligera tendencia hacia el incremento en el último año. La extracción de este producto en la región se inicia a principios del siglo (Padoch, 1990).

La extracción de otros productos, especialmente de plantas medicinales, es otro rubro que últimamente está en aumento; en las estadísticas aparece como plantas medicinales y cortezas. El potencial de estos productos en los bosques es muy importante, por lo que existe la tendencia a incrementarse en los próximos años.

\subsection{Procedimientos de extracción de los productos forestales diferentes de la madera}

El modo de extracción de los productos forestales diferentes de la madera es distinto al sistema que se emplea para la extracción de productos maderables, inclusive las herramientas que se utilizan son diferentes.

Este procedimiento es muy variado, dependiendo de diversos factores como especie, época del año, lugar y parte de la planta que se aprovecha.

En algunos casos se talan las plantas como por ejemplo en irapay, bombonaje, huambé y palmito; otras veces se aprovechan sin destruirlas como en shapaja, yarina, chambira, piasaba, aguaje y ungurahui, donde no es necesario talar los árboles. En el caso del ojé, lo que se aprovecha es el látex y para extraerlo no es necesario abatir la planta, solamente rasquetear la corteza alrededor del tallo.

Sin embargo, algunos extractores inescrupulosos talan las plantas sin discriminación de ninguna clase, con el objeto de aprovechar la mayor cantidad posible del producto, a pesar de reconocer los efectos negativos que conlleva este sistema en la utilización del bosque. 


\section{CONCLUSIONES}

Los productos forestales diferentes de la madera que se extraen en el área de influencia de Iquitos, son muy diversos; en este caso registramos 62 especies diferentes que proveen hojas, frutas, semillas, látex, fibras, raíces y otros productos.

El $37 \%$ de las especies anotadas proporciona alimentos en forma de frutas y semillas comestibles o de hojas tiernas, el $27 \%$ provee medicinas en forma de cortezas, raíces, hojas y exudados, mientras el $15 \%$ suministra materias primas para artesanías. Asimismo, el $21 \%$ se destina para otros usos, ya sea para construcción de viviendas, canoas, etc. Cerca de ocho especies tienen hasta dos usos cada una.

El $20 \%$ de las especies tratadas se extrae con el control del sector agrario, el resto se extrae clandestinamente; asimismo, el $10 \%$ de las especies reportadas procede de cultivos antrópicos.

El sector agrario registró en el período evaluado un total de 180 extractores. Este escaso número revela la existencia de una actividad altamente informal.

El mayor número de extractores se dedica al aprovechamiento de irapay, representando el $54,4 \%$.

La mayor parte de los productos extraídos del bosque procede de poblaciones silvestres cercanas a poblados asentados en las márgenes de los ríos Amazonas, Nanay y sus tributarios.

Las especies que crecen formando gremios o «manchales» son más propensas al aprovechamiento intenso, por los volúmenes en que se encuentran.

La especie con mayor volumen controlada por la Unidad Forestal en el periodo evaluado fue el palmito de huasaí, con $138205 \mathrm{~kg}$.

La extracción de los productos forestales diferentes de la madera se realiza de diversas formas, dependiendo de la especie, usos, época del año, lugar y parte de la planta que se aprovecha. 


\section{BIBLIOGRAFIA}

MEJIA, K. 1992. Las palmeras en los mercados de Iquitos. En: Bull de l' institut francais d' etudes andines 2 1(2): 755-769.

MEJIA, K. 1995. Diagnóstico de los recursos vegetales de la Amazonía Peruana. Documento técnico $\mathrm{N}^{\mathrm{o}}$ 16. Iquitos (Perú): IIAP. $59 \mathrm{pp}$.

MEJIA, K. y RENGIFO, E. 1995. Plantas medicinales de uso popular en la Amazonía Peruana. Lima: AECI .IIAP. Perú. 249 pp.

PADOCH, CH. 1990. «Importancia económica y comercialización de los productos del bosque y de las purmas en la región Iquitos». En: Denevan y Padoch editores. Agroforestería tradicional en la Amazonía Peruana (Documento $\mathrm{N}^{\mathrm{o}} 11,238$ p.). CIPA (160-193).

TELlO, R. et al. 1990. Diagnóstico socio-económico de la Región del Amazonas. Iquitos (Perú): Gobierno Regional del Amazonas. 205 pp. 


\section{Cuadro 1. Especies de productos forestales diferentes de la madera}

\begin{tabular}{|c|c|c|c|}
\hline NOM. COMUN & NOMBRE CIENTÍFICO & FAMILIA & USOS \\
\hline Abuta & Abutagrandifolia (C. Martius Sandwith & Menispermaceae & Relajante del sistema muscular. \\
\hline Aguaje & Mauritia Fleuxosa L.f & Arecaceae & $\begin{array}{l}\text { Fruto comestible, sirve para la elaboración de refrescos } \\
\text { (aguajina), helados chupetes, curichis; de los peciolos se } \\
\text { preparan esteras para interiores, faroles y veladores; del } \\
\text { fuste caldo se extrae el suri, que es consumido en su estado } \\
\text { larval, sea crudo, cocido o frito; las hojas sirven como } \\
\text { techos en algunos lugares; y otras múltiples aplicaciones. }\end{array}$ \\
\hline Aguajillo & Mauritiella aculeata (H. B. K.) Burret & Arecaceae & $\begin{array}{l}\text { Fruto comestible, cl tallo se usa partido para pisos y } \\
\text { divisiones en las casas rusticas. }\end{array}$ \\
\hline Almendro & Caryocar glabrum (Aublet) Persoon & Caryocariaceae & Fruto Comestible \\
\hline Ampihuasca & Chondrodendron tomentosum R. \& P. & Menispermaceae & $\begin{array}{l}\text { Una vez cocido y machacado los salios, se extrae un } \\
\text { poderoso veneno para envenenar la punta de flechas y } \\
\text { virotes; actualmente se usa en medicina como relajante } \\
\text { muscular. }\end{array}$ \\
\hline Apacharama & Licania britteniana Fritsch & Chrysobalanaceae & $\begin{array}{l}\text { La ceniza de la corteza se usa para dar consistencia a la } \\
\text { arcilla de las cerámicas. }\end{array}$ \\
\hline Ayahuasca & Banisterioopsis caapi (Spruee ex Grisebach) Morton & Malpighiaceae & $\begin{array}{l}\text { Los tallos son usados para preparar la «pulpa» de } \\
\text { «ayahuasca»» que es una bebida altamente alucinógena y } \\
\text { es un efectivo laxante; además se le atribuye propiedades } \\
\text { curativas dc cualquier enfermedad, adivinatorias y } \\
\text { telepáticas. }\end{array}$ \\
\hline Ayahuama & Couropita Subsessilis Pilger & Leeythidaceae & $\begin{array}{l}\text { Los Frutos sirven para alimentar gallinas, las yemas foliares } \\
\text { son utilizadas para calmar el dolor de dientes y el fruto - } \\
\text { también se usa para alejar el maleficio a través de } \\
\text { sahumerios. }\end{array}$ \\
\hline
\end{tabular}




\section{Continuación...}

\begin{tabular}{|c|c|c|c|}
\hline NOM. COMUN & NOMBRE CIENTÍFICO & FAMILIA & USOS \\
\hline Barbasco & Lonchocarpus nicou (Aublet) DC. & Fabaceae & Es un ictiotóxico utilizado para la pesca \\
\hline Bijao & Calathea lutea (Aublet) G. Meyer & Marantaceae & $\begin{array}{l}\text { La hoja sirve para proteger alimentos como para cocerlos y } \\
\text { usarlos, en juanes, tamales, patarashca, ninajuanes y otros. }\end{array}$ \\
\hline Bombonaje & Carludivica palmata $\mathrm{R} \& \mathrm{P}$ & Cyclanthaceae & $\begin{array}{l}\text { La raíz cocinada se contra vómitos y diarreas } \\
\text { De las hojas jóvenes se obtiene una fibra muy } \\
\text { resistente que se usa para manufacturar sombreros, } \\
\text { abanicos, cestas, cortinas y otros. Las hojas tiernas son } \\
\text { comestibles y la planta también se utiliza como } \\
\text { ornamento. }\end{array}$ \\
\hline Camu Camu & Myrciaria dubbia ( H. B. K) Mc Vaugh & Myrtaceae & $\begin{array}{l}\text { Frutos comestibles; en refrescos, la pulpa tiene un alto } \\
\text { contenido en ácido ascórbico. }\end{array}$ \\
\hline Canela moena & Endlicheria anomala (Nees) Mez & Lauraceae & Madera para canoas \\
\hline Caña brava & Gynerium sagittatum (Aulblet) P. Beauvois & Poaceae & $\begin{array}{l}\text { Los tallos son usados en la construcción y acabado de } \\
\text { viviendas, los tallos frescos se usan en cercos de huertos, se. } \\
\text { emplean para manufacturar flechas y para pesca; también } \\
\text { para hacer diversos artículos dc artesanía y las hojas sirven } \\
\text { para el tratamiento de procesos asmáticos. }\end{array}$ \\
\hline Cascarilla & Ladembergia magnifolia (R. \& P) Klotzsch & Rubiaceae & La infusión de la corteza de bebe para tratar el paludismo \\
\hline Castaña & Bertholletia excelsa Humboldt \& Bonpland & Lecythidaceae & $\begin{array}{l}\text { Semillas comestibles, contienen alto porcentaje de aceite } \\
\text { usado en la alimentación y para la fabricación de jabón. }\end{array}$ \\
\hline Caucho & Hevea brasiliensis (Willdenow ex Adr. Jussieu) Muell .Arg. & Euphorbiaceae & $\begin{array}{l}\text { Del látex se extrae una goma homogénea, usada en la } \\
\text { industria. }\end{array}$ \\
\hline
\end{tabular}


Continuación...

\begin{tabular}{|c|c|c|c|}
\hline NOM. COMUN & NOMBRE CIENTÍFICO & FAMILIA & USOS \\
\hline Cinamillo & Oenocarpus mapora Karsten & Arecaceae & $\begin{array}{l}\text { Los frutos puestos al calor solar o en agua caliente liberan el } \\
\text { mesocarpo, del que se prepara una bebida agradable y } \\
\text { refrescante; la hojas tiernas son comestibles y las maduras } \\
\text { se usan para techar casas. }\end{array}$ \\
\hline Clavo huasca & Tynanthus panurensis (Bureau) Sandwith & Bignoniaceae & $\begin{array}{l}\text { Los tallos cortados en trozos y macerados en aguardiente se } \\
\text { usan como bebida espirituosa. estimulante y para el } \\
\text { reumatismo }\end{array}$ \\
\hline Copaiba & Copaifera paupera (Herzog) Dwyer & Fabaceae & El aceite se usa como linimento medicina. \\
\hline Copal & Dacryodes peruviana (Loesener) J. F Macbride & Burseraceae & $\begin{array}{l}\text { El látex se usa junto con el de otros copales para calafatear } \\
\text { botes. }\end{array}$ \\
\hline Chambira & Astrocarrym chambira Burret & Arecaceae & $\begin{array}{l}\text { Los frutos tienen semillas comestibles. De las hojas jóvenes } \\
\text { se extrae una fibra que se usa para la manufactura de } \\
\text { hamacas, bolsas y toda clase de manualidades donde se } \\
\text { emplea hilo y cordel. }\end{array}$ \\
\hline Charichuelo & Rheedia benthamiana Planchon \& Triana & Clusiaceae & Frutos comestibles \\
\hline Chiele huayo & Lacmellea floribunda (Poepping) Bentham & Apocinaceae & Frutos comestibles \\
\hline Chimicua & Perebea sp. & & $\begin{array}{l}\text { Madera para plantillas de botes. El látex es osado como } \\
\text { pectoral y cicatrizante de heridas recientes; también como } \\
\text { tónico contra la debilidad general y como emplasto en las } \\
\text { contusiones. }\end{array}$ \\
\hline
\end{tabular}




\begin{tabular}{|c|c|c|c|}
\hline NOM. COMUN & NOMBRE CIENTÍFICO & FAMILIA & USOS \\
\hline Chopé & Gustavia longifolia Poeppig ex Berg & Lecythidaceae & Frutos comestibles, también se usa como ornamento. \\
\hline Chuchuhuasi & $\begin{array}{l}\text { Heisteria acuminata (Humbodt \& } \\
\text { Bonpland) Engler }\end{array}$ & Olacaceae & $\begin{array}{l}\text { A la maceración alcohólica de la corteza se le atribuyen varias propiedades } \\
\text { curativas, entre ellas antirreumática, antiartrítica, antidiarreica, para desarreglos } \\
\text { menstruales y estomacales, pero el uso más difundido es como bebida espirituosa. }\end{array}$ \\
\hline Gramalote & Hymenachne danacifolia (Raddi) Chase & Poaceae & Utilizada en artesanía \\
\hline Huacapurana & $\begin{array}{l}\text { Campsiandra angustifolia Spruce ex } \\
\text { Bentham }\end{array}$ & Leguminosae & $\begin{array}{l}\text { La corteza macerada en aguardiente se usa en la Amazonía Peruana como } \\
\text { antirreumático. }\end{array}$ \\
\hline Huambé & Philodendron sp. & Araceae & $\begin{array}{l}\text { El tallo de la liana se usa para manufacturar cestas, carteras, artesanías, en el } \\
\text { acabado de sillas y Otros muebles; la exudación de las hojas se usa para extraer } \\
\text { gusanos dc la piel. }\end{array}$ \\
\hline Huayruro & $\begin{array}{l}\text { Ormosia coccinea (Spruce ex Bentham) } \\
\text { Rudd }\end{array}$ & Fabaceae & Las semillas se usan en artesanía para collares, chaquiras y otros. \\
\hline
\end{tabular}




\section{Continuación...}

\begin{tabular}{|c|c|c|c|}
\hline NOM. COMUN & NOMBRE CIENTÍFICO & FAMILIA & USOS \\
\hline Huicungo & Astrocaryum macrocalyx Burret & Arecaceae & $\begin{array}{l}\text { Los frutos sois comestibles y las hojas se empiezan de vez en cuando para techos } \\
\text { provisionales luego de sacarles las espinas. }\end{array}$ \\
\hline Huimba & $\begin{array}{l}\text { Ceiba samauma (C. Martius \& Zuccarini) } \\
\text { Schumann }\end{array}$ & Bombacaceae & $\begin{array}{l}\text { La madera se usa para boyas en el transporte de otras maderas y para balsas; el vilano } \\
\text { de las semillas es usado por los nativos en sus virotes. }\end{array}$ \\
\hline Huito & Genipa americaca L & Rubiacaceae & $\begin{array}{l}\text { Los frutos maduros son comestibles al natural para combatir la bronquitis, el fruto } \\
\text { verde es usado por los nativos para teñir sus vestimentas y rostros, los frutos y } \\
\text { semillas hervidos sirven para combatir las inflamaciones del aparato genital } \\
\text { femenino. Algunas personas afirman que la decocción del fruto verde es abortiva. }\end{array}$ \\
\hline Ipururo & $\begin{array}{l}\text { Alchornea castaneifolia (Willdenow) } \\
\text { Adr.Jussieu }\end{array}$ & Euphorbiaceae & $\begin{array}{l}\text { La maceración alcohólica de la corteza se emplea en el tratamiento del reumatismo, } \\
\text { artritis, resfrió y dolores de los miembros causados por las faenas de pesca. }\end{array}$ \\
\hline Irapay & Lepidocaryum tenue C. Martius & Arecaceae & $\begin{array}{l}\text { Las hojas se utilizan para confeccionar las «crisnejas» (conjunto de hojas } \\
\text { entrelazadas entre si y amarradas a una ripa de tallo de Socratea sp.). las que se } \\
\text { emplean para techar casas. }\end{array}$ \\
\hline Leche caspi & Couma macrocarpa Barbosa Rodríguez & Apocinaceae & $\begin{array}{l}\text { El látex es usado para la fabricación dc chicles y también se usa para combatir la } \\
\text { diarrea por amebas y asma; aplicado localmente mejora la piel irritada por cl «arco»; } \\
\text { los frutos son comestibles. }\end{array}$ \\
\hline
\end{tabular}




\section{Continuación....}

\begin{tabular}{|c|c|c|c|}
\hline NOM. COMUN & NOMBRE CIENTÍFICO & FAMILIA & USOS \\
\hline Llanchama & Poulsenia armata (Miquel) Standley & Moraceae & La corteza convenientemente $<<$ batida $>>$ es usada en artesanía. \\
\hline Macambo & $\begin{array}{l}\text { Theobroma bicolor Humboldt \& } \\
\text { Bonpland }\end{array}$ & Sterculiaceae & Frutos comestibles \\
\hline Ojé & Ficus Insípida Willdenow & Moraceae & El látex es usado como vermífugo y para combatir los parásitos intestinales. \\
\hline Palillo & Campomanesia lineatifolia R. \& P. & Myrtaceae & $\begin{array}{l}\text { Fruto comestible; la raspadura de madera en agua se usa para pociones contra la } \\
\text { hemoptisis. }\end{array}$ \\
\hline Palmito & Euterpe oleracea C. Martius & Arecaceae & Como planta ornamental. Las hojas tiernas son comestibles. \\
\hline Paisaba & $\begin{array}{l}\text { Aphandra natalia (Balslev \& Henderson ) } \\
\text { Barford }\end{array}$ & Arecaceae & $\begin{array}{l}\text { Produce una fibra usada para cepillos y escobas, las hojas se usan para techar casas y } \\
\text { las semillas son comestibles. }\end{array}$ \\
\hline Pijuayo & Bactris gasipaes H.B.K & Arecaceae & $\begin{array}{l}\text { Fruto y semilla comestibles; de los frutos cocidos se prepara la bebida regional }<< \\
\text { masato }>>\text { y también se extrae aceite en forma domestica. Las yemas foliares son } \\
\text { comestibles al natural o en ensaladas. }\end{array}$ \\
\hline Pona & $\begin{array}{l}\text { Socratea axorrhiza (C. Martius) H.A } \\
\text { Wendland }\end{array}$ & Arecaceae & $\begin{array}{l}\text { Es usado para pesos, paredes, divisiones de habitaciones, para tejer las }<<\text { crisnejas }>> \\
\text { de la hoja }<<\text { irapay }>>\text {, entre otros. }\end{array}$ \\
\hline
\end{tabular}




\begin{tabular}{|c|c|c|c|}
\hline NOM. COMUN & NOMBRE CIENTÍFICO & FAMILIA & USOS \\
\hline Remo caspi & Aspidosperma excelsum Bentham & Apocinaceae & Se utiliza para la confección de remos. \\
\hline Renaco & Ficus sp. & Moraceae & Es usado como antidiarreico y como remedio contra las heridas infectadas. \\
\hline Sachamango & Grias neuberthii J.F Macbride & Lecythidaceae & Frutos comestibles y como ornamento. \\
\hline S. de grado & Croton lechleri Muell. Arg. & Euphorbiaceae & $\begin{array}{l}\text { El látex se usa para cicatrizar heridas, en lavados vaginales post parto y también se } \\
\text { recomienda para las úlceras estomacales e intestinales. }\end{array}$ \\
\hline Shapaja & $\begin{array}{l}\text { Sceelea phalerata (C. Martius ex } \\
\text { Sprengel) Burret }\end{array}$ & Arecaceae & $\begin{array}{l}\text { Es utilizado para cl techado; el endospermo inmaduro, las hojas tiernas y parte del } \\
\text { estípite blando son comestibles. También sirve para confeccionar cumbas para el } \\
\text { techado de casas y otros usos. }\end{array}$ \\
\hline Shimbillo & Inga sp. & Fabaceae & Frutos comestibles \\
\hline Tamamuri & Brosimun acutifolium Huber & Moraceae & El tronco y la corteza se usan para combatir el reumatismo. \\
\hline Tamshi & Heteropsis sp. & Fabaceae & $\begin{array}{l}\text { Las ralees se usan en artesanía y también las lianas, aunque éstas preferentemente } \\
\text { para canastas y también en ataduras, principalmente en la construcción de casas } \\
\text { rústicas. }\end{array}$ \\
\hline Topa & $\begin{array}{l}\text { Ochroma pyramidale (Cavanilles ex } \\
\text { Lamarck) Urban. }\end{array}$ & Bombacaceae & $\begin{array}{l}\text { La madera se usa para balsas, casas flotantes, artesanías, boyas para madera, } \\
\text { flotadores dc redes y anzuelos; la Corteza se emplea en cordelería; al vilano de las } \\
\text { semillas se le denomina «flor de topan, la que se emplea en colchonería y como } \\
\text { relleno de juguetes. }\end{array}$ \\
\hline
\end{tabular}




\begin{tabular}{|c|c|c|c|}
\hline NOM. COMUN & NOMBRE CIENTÍFICO & FAMILIA & USOS \\
\hline Ubos & Spondias mombin L. & Anacardiaceae & $\begin{array}{l}\text { Los frutos son comestibles y son usados para combatir la diarrea, la TBC y como } \\
\text { reconstituyente para después dcl parto. }\end{array}$ \\
\hline Ungurahui & Oenocarpus batana C. Martius & Arecaceae & $\begin{array}{l}\text { Los frutos son comestibles al natural, en refrescos, helados, chupetes; las hojas se } \\
\text { usan para techar casas; de los frutos maduros se extrae en forma artesanal un fino } \\
\text { aceite como tónico para el cabello y usado como linimento y laxante. El tallo también } \\
\text { se emplea en la construcción de viviendas rurales. }\end{array}$ \\
\hline Uña de gato & $\begin{array}{l}\text { Uncaria guianensis (Aublet Gmelia } \\
\text { U. Tomentosa (Willdenow ex } \\
\text { Roemer \& Schultes) DC. }\end{array}$ & Rubiaceae & $\begin{array}{l}\text { La infusión de las hojas es usada como antigripal y para el tratamiento dcl cáncer; la } \\
\text { corteza es utilizada en infusiones para reforzar cl sistema inmunológico. }\end{array}$ \\
\hline Varacasha & Desmoncus mitis C. Martius & Arecaceae & $\begin{array}{l}\text { Los tallos partidos y pulidos se usan para manufacturar cestas, canastas, esterillas de } \\
\text { sillas y otros artículos de artesanía. }\end{array}$ \\
\hline Yarina & Phytelepas macrocarpa R. \& P. & Arecaceae & $\begin{array}{l}\text { Las hojas maduras se usan para techar casas, mientras que las hojas tiernas y el } \\
\text { endospermo inmaduro son comestibles. }\end{array}$ \\
\hline Yuquilla & Euphorbia cotinifolia & Euphorbiaceae & Como plantas ornamentales, el látex es cáustico, ictiotóxico e insecticida. \\
\hline
\end{tabular}

